\title{
Karyotypic variation and geographic distribution of Anopheles campestris-like (Diptera: Culicidae) in Thailand
}

\author{
Sorawat Thongsahuan', Visut Baimai², Yasushi Otsuka3, Atiporn Saeung1, Benjawan Tuetun', \\ Narissara Jariyapan', Sittiporn Suwannamit', Pradya Somboon', Atchariya Jitpakdi', \\ Hiroyuki Takaoka ${ }^{3}$, Wej Choochote ${ }^{1 /+}$
}

\begin{abstract}
${ }^{1}$ Department of Parasitology, Faculty of Medicine, Chiang Mai University, 50200 Chiang Mai, Thailand ${ }^{2}$ Department of Biology and Centre for Vectors and Vector-Borne Diseases, Faculty of Science, Mahidol University, Bangkok, Thailand ${ }^{3}$ Department of Infectious Disease Control, Faculty of Medicine, Oita University, Hasama, Oita, Japan
\end{abstract}

Seventy-one isolines of Anopheles campestris-like were established from wild-caught females collected from human-biting and animal-biting traps at 12 locations in Thailand. All isolines had an average branch summation of seta 2-VI pupal skins ranging from 20.3-30.0 branches, which is in the range of An. campestris (17-58 branches). They showed three different karyotypes based on the amount of extra heterochromatin in the sex chromosomes, namely Forms $B\left(X_{2}, Y_{2}\right), E\left(X_{1}, X_{2}, X_{3}, Y_{s}\right)$ and a new karyotypic Form $F\left(X_{2}, X_{3}, Y_{6}\right)$. Form $B$ has been found only in Chaing Mai and Kamphaeng Phet populations, while Forms $E$ and $F$ are widely distributed throughout the species range. Genetic crosses between the 12 isolines, which were arbitrarily selected as representatives of An. campestrislike Forms $B, E$ and $F$, revealed genetic compatibility that provided viable progeny through $F$ generations, suggesting a conspecific nature of these karyotypic forms. These results are supported by the very low intraspecies variation (genetic distance < 0.005) of ITS2, COI and COII from genomic DNA of the three karyotypic forms.

Key words: Anopheles campestris-like - metaphase karyotype - crossing experiment - ITS2 - COI - COII

Anopheles (Anopheles) barbirostris belongs to the Barbirostris Subgroup of the Myzorhynchus Series and is widely distributed in Thailand and Southeast Asia (Reid 1968, Scanlon et al. 1968, Harrison 1980, Harbach 2004, Rattanarithikul et al. 2006). Normally, An. barbirostris and the closely related species, Anopheles campestris, can cause problems in species identification because of their similarity in external morphology. Accordingly, An. barbirostris was formerly considered a suspected vector of malaria and/or filariasis in Thailand (Iyengar 1953, Griffith 1955), while it has been incriminated as a natural vector of Plasmodium vivax and Brugia malayi, the causative agent of filariasis, in Indonesia (Atomosoedjono et al. 1976, Kirnowardoyo 1985). Recently, mosquitoes of the anthropophilic An. barbirostris/campestris complex were incriminated as potential natural vectors of $P$. vivax in the Aranyaprathet district, $\mathrm{Sa}$ Kaeo province (Limrat et al. 2001). Mosquitoes of this complex have played an important role in increasing cases of $P$. vivax infection in Thailand (Sattabongkot et al. 2004). Recent morphological, cytological, hybridization and molecular analysis have revealed that An. campestris-like and An.

Financial support: Biodiversity Research and Training Program (BRT R_250009), Thailand Research Fund through the Royal Golden Jubilee PhD Program (PHD/0082/2549), Faculty of Medicine Endowment Fund, Chiang Mai University, Thailand

+ Corresponding author: wchoocho@mail.med.cmu.ac.th

Received 30 January 2009

Accepted 30 June 2009 barbirostris are distinct species (Saeung et al. 2007). Furthermore, similar studies have shown that An. barbirostris s.1. is a cryptic species consisting of at least four sibling species, i.e., A1, A2, A3 (Saeung et al. 2008) and A4 (Suwannamit et al. 2009). The An. barbirostris species complex exhibited karyotypic variation due to different amounts of extra heterochromatin in the sex chromosomes. Likewise, our previous observations indicated that An. campestris-like had at least two karyotypic forms, i.e., Forms $\mathrm{B}\left(\mathrm{X}_{2}, \mathrm{Y}_{2}\right)$ and $\mathrm{E}\left(\mathrm{X}_{2}, \mathrm{Y}_{5}\right)$ (Saeung et al. 2007). Thus, it has been suggested that the acquisition of extra block(s) of heterochromatin played an important role in the chromosomal evolution of Oriental Anopheles (Baimai 1998). The crossing experiments between isolines of An. campestris-like Forms B and E showed no post-mating reproductive isolation. Comparative studies of nucleotide sequences of rDNA of ITS2 and mtDNA of COI and COII, among the isolines of An. campestris-like Forms B and E, revealed nearly identical and/or very low intraspecific variation (genetic distance $<0.005$ ) (Saeung et al. 2007). Thus, crossing and molecular evidence support the conspecific relationships of the karyotypic forms of An. campestris-like mosquitoes.

This paper describes a new karyotypic form of $A n$. campestris-like. We also present the results of crossing experiments and comparative DNA sequencing of the ITS2, COI and COII regions of the three karyotypic forms of An. campestris-like in Thailand.

\section{MATERIALS AND METHODS}

Field collections and establishment of isoline colonies - Wild-caught, fully engorged female mosquitoes of $A n$. campestris-like were collected from human-baited and 
buffalo-baited traps during September 2006-December 2007 at 12 localities in Thailand (Fig. 1, Table I). A total of 71 isolines were successfully established and maintained in our insectary using the techniques described by Choochote et al. (1983) and Kim et al. (2003). These isolines were used for studies on metaphase karyotype, crossing experiments and molecular analysis.

Metaphase karyotype - Metaphase chromosomes were prepared from 10 samples of the early fourth-instar larval brains of $F_{1}$ and/or $F_{2}$-progenies of each isoline using the techniques previously described by Saeung et al. (2007, 2008). Identification of karyotypic forms followed the cytotaxonomic key of Baimai et al. (1995).

Crossing experiments - The 12 laboratory-raised isolines of An. campestris-like were arbitrarily selected from the 30 isoline colonies as representatives of the three karyotypic forms, i.e., Form B (AKpB1), Form E (HCE6, AKkE4, AMkE1, AMsE3, HSkE3, ACpE6) and Form F (AUdF5, ACiF1, AAyF2, HCtF4, APkF1) (Table II). These isolines were used for crossing experiments, in order to determine post-mating reproductive isolation by employing the techniques previously reported by Saeung et al. $(2007,2008)$.

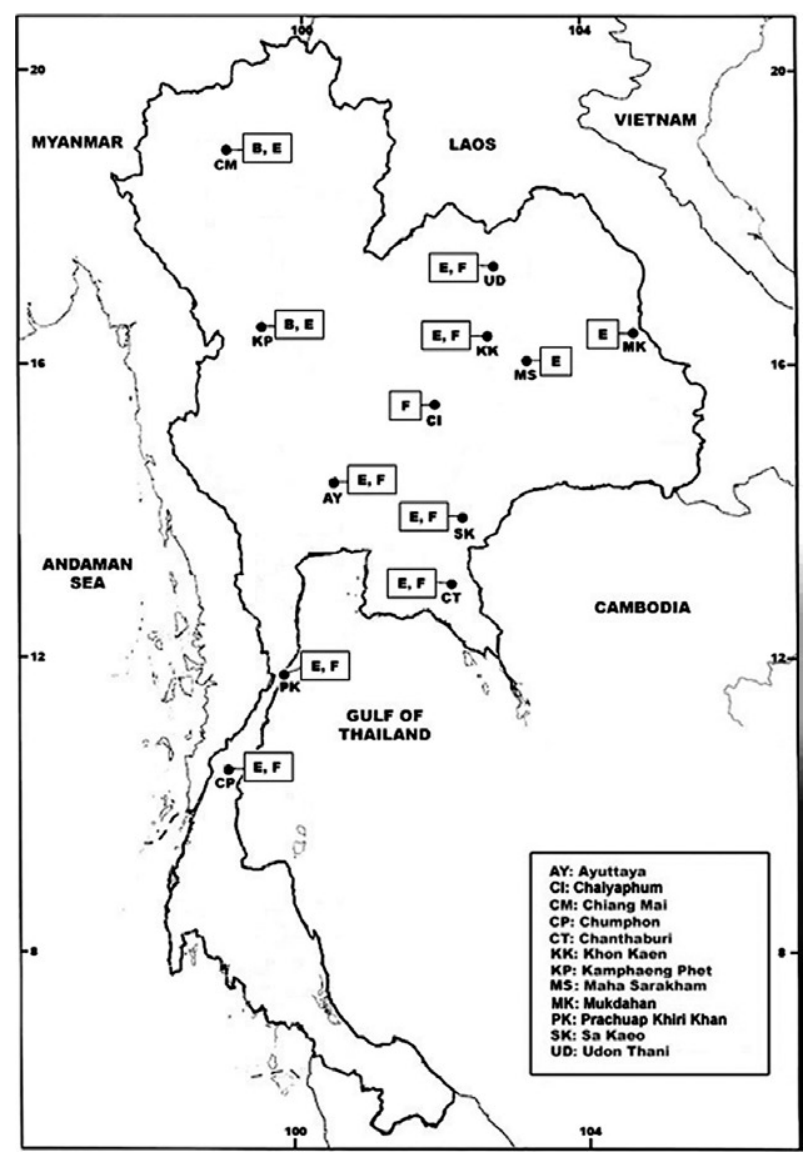

Fig. 1: map of Thailand showing distribution of Anopheles campestris-like Form B, E and F.
DNA extraction, amplification and sequencing - Individual feral and/or $\mathrm{F}_{1}$-progeny adult females of each isoline were used for DNA extraction and amplification. Genomic DNA was extracted from a whole adult mosquito using a DNeasy ${ }^{\circledR}$ Blood \& Tissue Kit (Qiagen) according to the manufacturer's instructions. The amplification was done with primers and conditions, as described previously (Saeung et al. 2007, 2008). The rDNA ITS2, COI and COII regions were amplified by polymerase chain reaction (PCR) using the following primers: ITS2A, 5' -TGTGAACTGCAGGACACAT-3' and ITS2B, 5' -TATGCTTAAATTCAGGGGGT-3' for rDNA ITS2; LCO1490 (f), 5' -GGTCAACAAATCATAAAGATATTGG-3' and HCO2198 (r), 5' -TAAACTTCAGGGTGACCAAAAAATCA-3' for COI; and LEU (f), 5' -TCTAATATGGCAGATTAGTGCA-3' and LYS (r), 5' -ACTTGCTTTCAGTCATCTAATG-3' for COII. PCR was carried out using $20 \mu \mathrm{L}$ volumes containing 0.5 units of Ex Taq (Takara), 1X Ex Taq buffer, $2 \mathrm{mM}$ of $\mathrm{MgCl}_{2}$, $0.2 \mathrm{mM}$ of each dNTP, $0.25 \mu \mathrm{M}$ of each primer and 1 $\mu \mathrm{L}$ of the extracted DNA. The amplified products were electrophorised through a $1 \%$ agarose gel. PCR products of ITS2 were gel purified with the QIAquick ${ }^{\circledR}$ Gel Extraction Kit (Qiagen) and cloned into pCR2.1-TOPO (Invitrogen). Sequences of several clones from each isoline were determined. PCR products of COI and COII were purified with the QIAquick ${ }^{\circledR}$ PCR Purification Kit (Qiagen) and directly sequenced. Sequencing reactions were performed using the BigDye ${ }^{\circledR}$ Terminator Cycle Sequencing Kit and run on a 3130 Genetic Analyzer (Applied Biosystems). The sequence data of this paper have been deposited in the DDBJ/EMBL/GenBank nucleotide sequence database under accession numbers AB436074-AB436157 (Table I).

DNA sequence and phylogenetic analysis - For the ITS2 DNA region, three individual clones from each isoline were sequenced and aligned using the CLUSTALW multiple alignment program (Thompson et al. 1994). Gap sites were excluded from the following analysis. Genetic distances were estimated using the Kimura two-parameter method (Kimura 1980). Construction of neighbour-joining trees (Saitou \& Nei 1987) and the bootstrap test, with 1,000 replications, were conducted using the MEGA version 4.0 program (Tamura et al. 2007) from the individual sequence of each isoline for all three DNA regions. The bootstrapping values, as percentages, are indicated above the branches of the tree. For the phylogenetic trees of COI and COII, Anopheles gambiae and Anopheles pullus were used as outgroups (NC_002084, AY444349, AY444350). The phylogenetic tree of ITS2 was constructed as an unrooted tree because an outgroup with easily aligned ITS2 was not available. The published data of An. campestris-like and An. barbirostris described by Saeung et al. $(2007,2008)$ were also used for phylogenetic analysis.

\section{RESULTS}

Morphological and karyotypic characters - Morphological investigations of $F_{1}$ and/or $F_{2}$-progenies of the 71 isolines showed an average summation of 20.3-30.0 seta 
TABLE I

Locations, isoline colonies and karyotypic forms of Anopheles campestris-like and their GenBank accession numbers

\begin{tabular}{|c|c|c|c|c|c|c|c|}
\hline \multirow{2}{*}{$\begin{array}{l}\text { Location } \\
\text { (geograpical } \\
\text { coordinates) }\end{array}$} & \multirow[b]{2}{*}{$\begin{array}{l}\text { Code of } \\
\text { isoline }^{a}\end{array}$} & \multirow[b]{2}{*}{$\begin{array}{l}\text { Karyotypic } \\
\text { form }\end{array}$} & \multirow[b]{2}{*}{$\begin{array}{l}\text { Length of } \\
\text { ITS2 (bp) }\end{array}$} & \multirow[b]{2}{*}{ Region } & \multicolumn{3}{|c|}{ Genbank accession number } \\
\hline & & & & & ITS2 & COI & COII \\
\hline \multirow[t]{7}{*}{$\begin{array}{l}\text { Chiang Mai } \\
\left(18^{\circ} 47^{\prime} \mathrm{N} 98^{\circ} 59^{\prime} \mathrm{E}\right)\end{array}$} & HCE6 $^{b}$ & $\mathrm{E}$ & 1,651 & ITS2, COI, COII & AB331566 & AB331583 & AB331604 \\
\hline & HCB9 & $\mathrm{B}$ & 1,651 & ITS2, COI, COII & AB331563 & AB331582 & AB331601 \\
\hline & HCmE12 & $\mathrm{E}$ & 1,651 & ITS2, COI, COII & AB436074 & AB436102 & AB436130 \\
\hline & HCmE14 & $\mathrm{E}$ & 1,651 & ITS2, COI, COII & AB436075 & AB436103 & AB436131 \\
\hline & HCmE15 & $\mathrm{E}$ & 1,651 & ITS2, COI, COII & AB436076 & AB436104 & AB436132 \\
\hline & $\mathrm{HCmB18}$ & $\mathrm{B}$ & 1,651 & ITS2, COI, COII & AB436077 & AB436105 & AB436133 \\
\hline & $\mathrm{HCmB} 20$ & $\mathrm{~B}$ & 1,651 & ITS2, COI, COII & AB436078 & AB436106 & AB436134 \\
\hline \multirow[t]{2}{*}{$\begin{array}{l}\text { Kamphaeng Phet } \\
\left(16^{\circ} 50^{\prime} \mathrm{N} 99^{\circ} 04^{\prime} \mathrm{E}\right)\end{array}$} & $\mathrm{AKpB} 1^{b}$ & $\mathrm{~B}$ & 1,651 & ITS2, COI, COII & AB436079 & AB436107 & AB436135 \\
\hline & HKpE1 & $\mathrm{E}$ & 1,651 & ITS2, COI, COII & AB436080 & AB436108 & AB436136 \\
\hline \multirow[t]{3}{*}{$\begin{array}{l}\text { Ayuttaya } \\
\left(14^{\circ} 01^{\prime} \mathrm{N} 101^{\circ} 02^{\prime} \mathrm{E}\right)\end{array}$} & $\mathrm{AAyF} 2^{b}$ & $\mathrm{~F}$ & 1,651 & ITS2, COI, COII & AB436081 & AB436109 & AB436137 \\
\hline & AAyF6 & $\mathrm{F}$ & 1,651 & ITS2, COI, COII & AB436082 & AB436110 & AB436138 \\
\hline & AAyE7 & $\mathrm{E}$ & 1,651 & ITS2, COI, COII & AB436083 & AB436111 & AB436139 \\
\hline \multirow[t]{3}{*}{$\begin{array}{l}\text { Udon Thani } \\
\left(17^{\circ} 24^{\prime} \mathrm{N} 102^{\circ} 47^{\prime} \mathrm{E}\right)\end{array}$} & AUdF3 & $\mathrm{F}$ & 1,651 & ITS2, COI, COII & AB436084 & AB436112 & AB436140 \\
\hline & AUdF4 & $\mathrm{F}$ & 1,651 & ITS2, COI, COII & AB436085 & AB436113 & AB436141 \\
\hline & $\mathrm{AUdF}^{b}$ & $\mathrm{~F}$ & 1,651 & ITS2, COI, COII & AB436086 & AB436114 & AB436142 \\
\hline \multirow[t]{3}{*}{$\begin{array}{l}\text { Khon Kaen } \\
\left(15^{\circ} 41^{\prime} \mathrm{N} 101^{\circ} 45^{\prime} \mathrm{E}\right)\end{array}$} & AKkF1 & $\mathrm{F}$ & 1,651 & ITS2, COI, COII & AB436087 & AB436115 & AB436143 \\
\hline & $\mathrm{AKkE} 4^{b}$ & $\mathrm{E}$ & 1,651 & ITS2, COI, COII & AB436088 & AB436116 & AB436144 \\
\hline & AKkE8 & $\mathrm{E}$ & 1,651 & ITS2, COI, COII & AB436089 & AB436117 & AB436145 \\
\hline \multirow[t]{3}{*}{$\begin{array}{l}\text { Maha Sarakham } \\
\left(15^{\circ} 45^{\prime} \mathrm{N} 103^{\circ} 01^{\prime} \mathrm{E}\right)\end{array}$} & $\mathrm{AMsE}^{b}$ & $\mathrm{E}$ & 1,651 & ITS2, COI, COII & AB436090 & AB436118 & AB436146 \\
\hline & AMsE4 & $\mathrm{E}$ & 1,651 & ITS2, COI, COII & AB436091 & AB436119 & AB436147 \\
\hline & AMsE5 & $\mathrm{E}$ & 1,651 & ITS2, COI, COII & AB436092 & AB436120 & AB436148 \\
\hline \multicolumn{7}{|l|}{ Mukdahan } & AB436149 \\
\hline $\begin{array}{l}\text { Chaiyaphum } \\
\left(15^{\circ} 481^{\prime} \mathrm{N} 101^{\circ} 30^{\prime} \mathrm{E}\right)\end{array}$ & $\mathrm{ACiF}^{b}$ & $\mathrm{~F}$ & 1,651 & ITS2, COI, COII & AB436094 & AB436122 & AB436150 \\
\hline \multirow[t]{3}{*}{$\begin{array}{l}\text { Sa Kaeo } \\
\left(13^{\circ} 14^{\prime} \text { N 10151'E) }\right.\end{array}$} & HSkF1 & $\mathrm{F}$ & 1,651 & ITS2, COI, COII & AB436095 & AB436123 & AB436151 \\
\hline & HSkE2 & $\mathrm{E}$ & 1,651 & ITS2, COI, COII & AB436096 & AB436124 & AB436152 \\
\hline & $\mathrm{HSkE}^{b}$ & $\mathrm{E}$ & 1,651 & ITS2, COI, COII & AB436097 & AB436125 & AB436153 \\
\hline \multirow[t]{2}{*}{$\begin{array}{l}\text { Chanthaburi } \\
\left(12^{\circ} 37^{\prime} \mathrm{N} 102^{\circ} 07^{\prime} \mathrm{E}\right)\end{array}$} & HCtE2 & $\mathrm{E}$ & 1,651 & ITS2, COI, COII & AB436098 & AB436126 & AB436154 \\
\hline & $\mathrm{HCtF} 4^{b}$ & $\mathrm{~F}$ & 1,651 & ITS2, COI, COII & AB436099 & AB436127 & AB436155 \\
\hline $\begin{array}{l}\text { Prachuap Khiri Khan } \\
\left(11^{\circ} 48^{\prime} \mathrm{N} 99^{\circ} 49^{\prime} \mathrm{E}\right)\end{array}$ & $\mathrm{APkF}^{b}$ & $\mathrm{~F}$ & 1,651 & ITS2, COI, COII & AB436100 & AB436128 & AB436156 \\
\hline $\begin{array}{l}\text { Chumphon } \\
\left(10^{\circ} 29^{\prime} \mathrm{N} 99^{\circ} 11^{\prime} \mathrm{E}\right)\end{array}$ & $\mathrm{ACpE}^{b}$ & $\mathrm{E}$ & 1,651 & ITS2, COI, COII & AB436101 & AB436129 & AB436157 \\
\hline
\end{tabular}

$a$ : code of isoline: A: animal bait; H: human bait; $b$ : used in crossing experiments.

2-VI branches, which is in the range of topotypic $A n$. campestris (17-58 branches). Cytogenetic observations of $\mathrm{F}_{1}$ and/or $\mathrm{F}_{2}$-progenies of these isolines demonstrated three forms of metaphase karyotypes, i.e., Forms B ( $\mathrm{X}_{2}$, $\left.\mathrm{Y}_{2}\right), \mathrm{E}\left(\mathrm{X}_{1}, \mathrm{X}_{2}, \mathrm{X}_{3}, \mathrm{Y}_{5}\right)$ and $\mathrm{F}\left(\mathrm{X}_{2}, \mathrm{X}_{3}, \mathrm{Y}_{6}\right)$ (Fig. 2). Form B has been detected only in Chiang Mai and Kamphaeng Phet. However, Forms E and F have been encountered in sympatry in most populations throughout Thailand (Fig. 1, Table I). Interestingly, the three karyotypic forms have been found in the mosquitoes collected from both 
human-baits and animal-baits. Thus, it seems that there is no preferential host for these karyotypic forms.

The new metaphase karyotype, Form F, had submetacentric $\mathrm{X}_{2}$ and $\mathrm{X}_{3}$ chromosomes resembling those of Forms B and E. Nevertheless, the $\mathrm{Y}_{6}$ chromosome had a large subtelocentric shape, which was quite different from the submetacentric $\mathrm{Y}_{2}$ and the small metacentric $\mathrm{Y}_{5}$ chromosomes of Forms B and E, respectively (Fig. 2).

Crossing experiments - Details of hatchability, pupation, emergence and adult sex-ratio of parental, reciprocal and $\mathrm{F}_{1}$-hybrid crosses among the 12 isolines of An. campestris-like Forms B, E and F are shown in Table II. All crosses yielded viable progeny through $\mathrm{F}_{2}$ generations. No evidence of genetic incompatibility and/or post-mating reproductive isolation was observed among these crosses.

DNA sequences and phylogenetic analysis - DNA sequences were determined and analyzed for the ITS2, COI and COII regions of the 30 isolines of An. campestrislike Forms B, E and F. They all showed the same length for the ITS2 (1,651 bp), COI (658 bp) and COII (685 bp). The length of the three DNA regions of An. campestrislike Forms B and E obtained in this study agreed with that of the previous report (Saeung et al. 2007). Form F also showed no difference in length of the three DNA regions. To reveal the evolutionary relationship among the three karyotypic forms, neighbour-joining trees were constructed (Figs 3-5). Obviously, the average genetic distances within and between the three karyotypic forms of An. campestris-like exhibited no significant differences (0.001-0.004) in the three DNA regions (Table III). Hence, the 30 isolines were placed within a cluster of $A n$. campestris-like. However, the trees for ITS2, COI and COII of these isolines of An. campestris-like Forms B, E and $\mathrm{F}$ were clearly different from those of the four sibling species of the An. barbirostris complex with strongly supported bootstrap probabilities (95-100\%) (Figs 3-5).
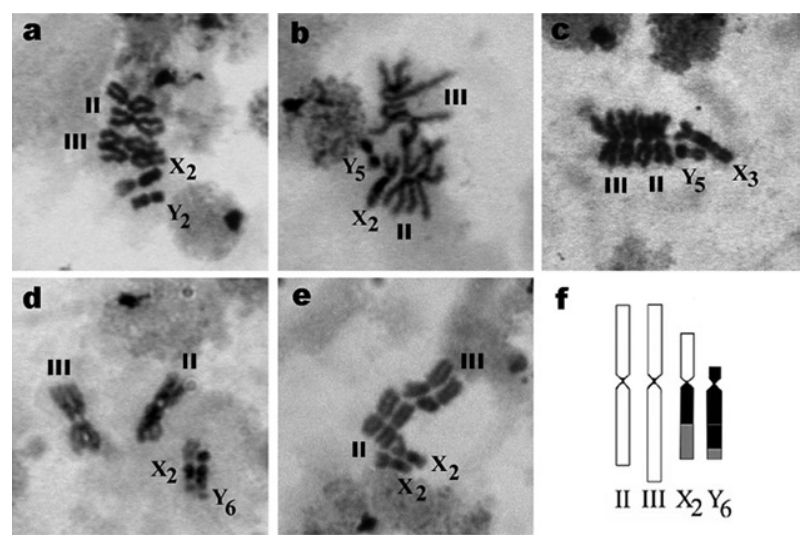

Fig. 2: metaphase karyotypes of Anopheles campestris-like Form B, $\mathrm{E}$ and $\mathrm{F}$ (a-e). Form B: a: Kamphaeng Phet strain, showing $\mathrm{X}_{2}, \mathrm{Y}_{2}$ chromosomes; Form E: b: Chumphon strain, showing $\mathrm{X}_{2}, \mathrm{Y}_{5}$ chromosomes; c: Sa Kaeo strain, showing $X_{3}, Y_{5}$ chromosomes; Form F: d: Udon Thani strain, showing $\mathrm{X}_{2}, \mathrm{Y}_{6}$ chromosomes; e: showing homozygous $\mathrm{X}_{2}, \mathrm{X}_{2}$ chromosomes; f: diagrams of representative metaphase karyotypes of An. campestris-like Form F.

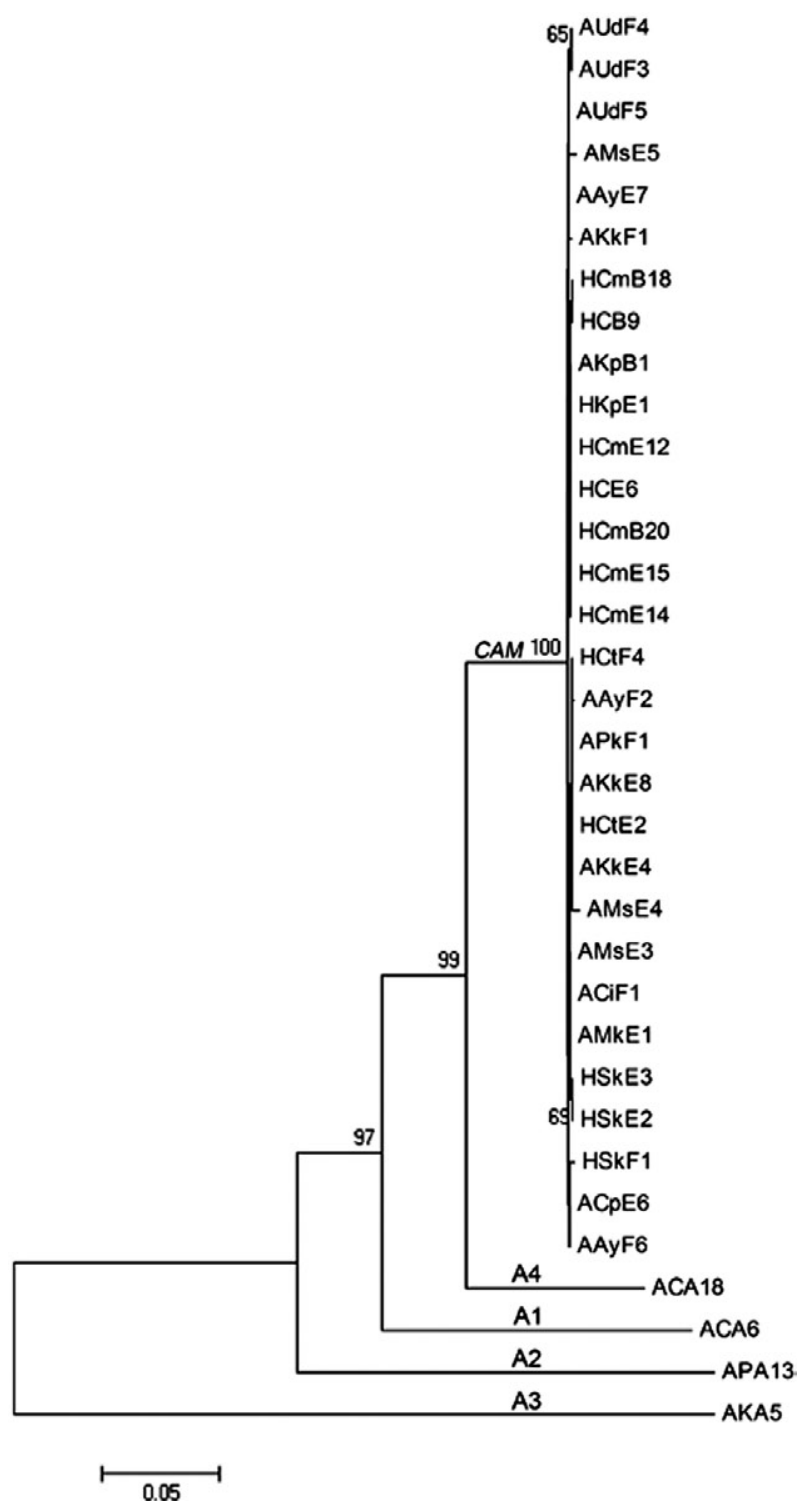

Fig. 3: a phylogenetic trees of Anopheles campestris-like Forms B, E and $\mathrm{F}(C A M)$ and Anopheles barbirostris species A1, A2, A3 and A4 based on molecular analysis of ITS2 sequences. The tree was generated by neighbor-joining analysis. Numbers on the nodes indicate probabilities based on 1,000 bootstrap replicates. A probability of more than $50 \%$ is shown. Branch lengths are proportional to genetic distance (scale bar).

\section{DISCUSSION}

Comparative studies on metaphase chromosomes of anopheline mosquitoes in Thailand revealed at least three karyotypic forms in An. barbirostris s.l., i.e., Forms A $\left(\mathrm{X}_{2}, \mathrm{X}_{3}, \mathrm{Y}_{1}\right), \mathrm{B}\left(\mathrm{X}_{1}, \mathrm{X}_{2}, \mathrm{X}_{3}, \mathrm{Y}_{2}\right)$ and $\mathrm{C}\left(\mathrm{X}_{2}, \mathrm{X}_{3}, \mathrm{Y}_{3}\right)$ and one karyotypic form in An. campestris s.l. (X, Y) (Baimai et al. 1995). Recently, Saeung et al. (2007) reported two and three karyotypic forms of An. campestris-like and the An. barbirostris species complex, respectively. Moreover, the two karyotypic forms of An. campestrislike were detected in Chiang Mai, i.e., Forms B $\left(\mathrm{X}_{2}\right.$, 


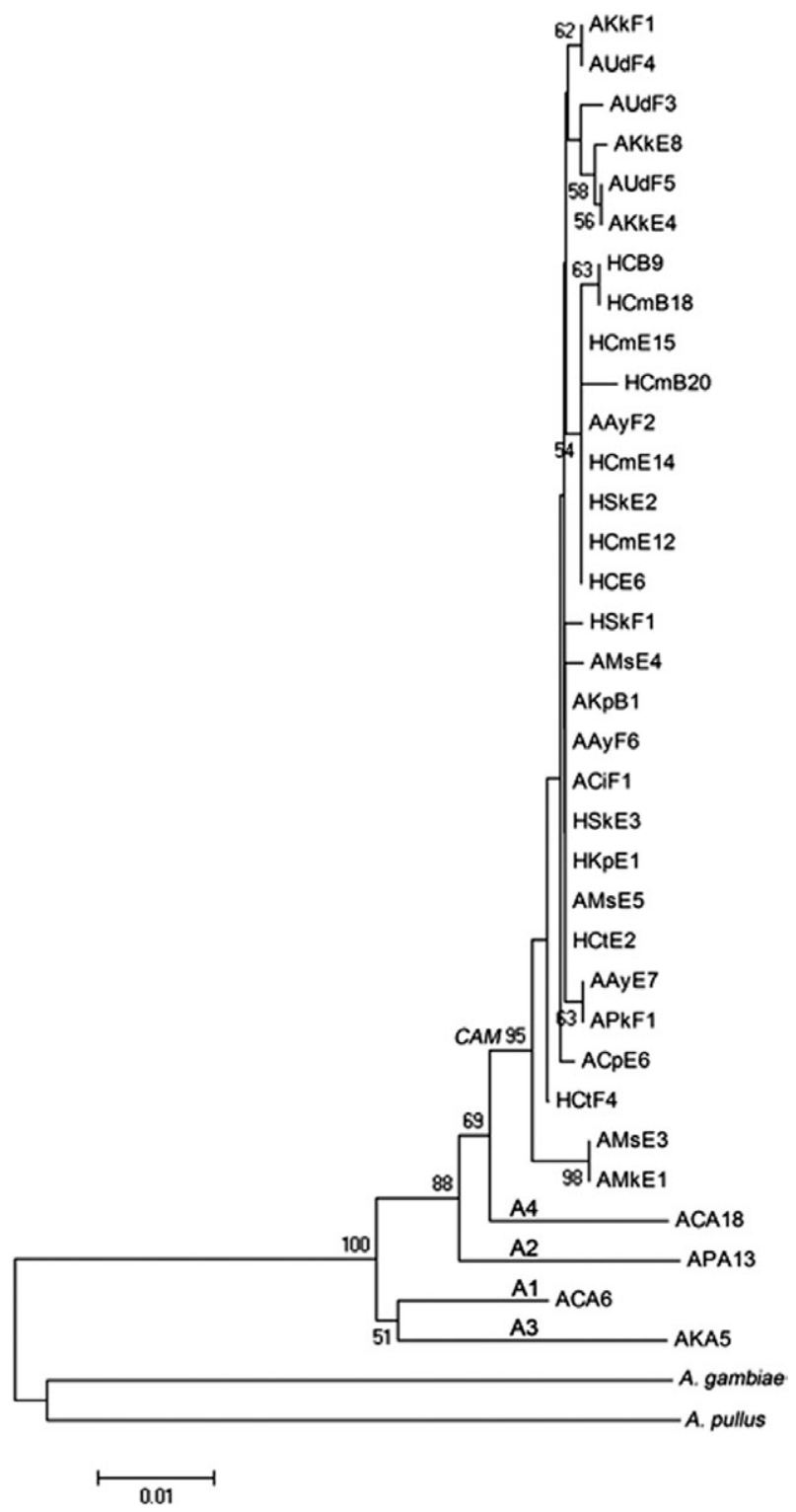

Fig. 4: a phylogenetic trees of Anopheles campestris-like Forms B, $\mathrm{E}$ and $\mathrm{F}(C A M)$ and Anopheles barbirostris species A1, A2, A3 and A4 based on molecular analysis of COI sequences. The tree was generated by neighbor-joining analysis. Numbers on the nodes indicate probabilities based on 1,000 bootstrap replicates. A probability of more than $50 \%$ is shown. Branch lengths are proportional to genetic distance (scale bar).

$\left.\mathrm{Y}_{2}\right)$ and $\mathrm{E}\left(\mathrm{X}_{2}, \mathrm{Y}_{5}\right)$. The $\mathrm{X}_{2}, \mathrm{X}_{3}, \mathrm{Y}_{6}$ chromosomes of $A n$. campestris-like were new discoveries in this study. Particularly, the $\mathrm{Y}_{6}$ chromosome was obviously different from the $\mathrm{Y}_{2}$ and $\mathrm{Y}_{5}$ chromosomes of An. campestris-like Forms $\mathrm{B}$ and $\mathrm{E}$ that were previously described.

Hybridization experiments for determining hybrid non-viability, sterility or breakdown are still useful criteria for biological species. Further, genetic incompatibility, including lack of insemination, embryonation, hatchability, larval survival, pupation, emergence, adult sex distortion, abnormal morphology and reproductive

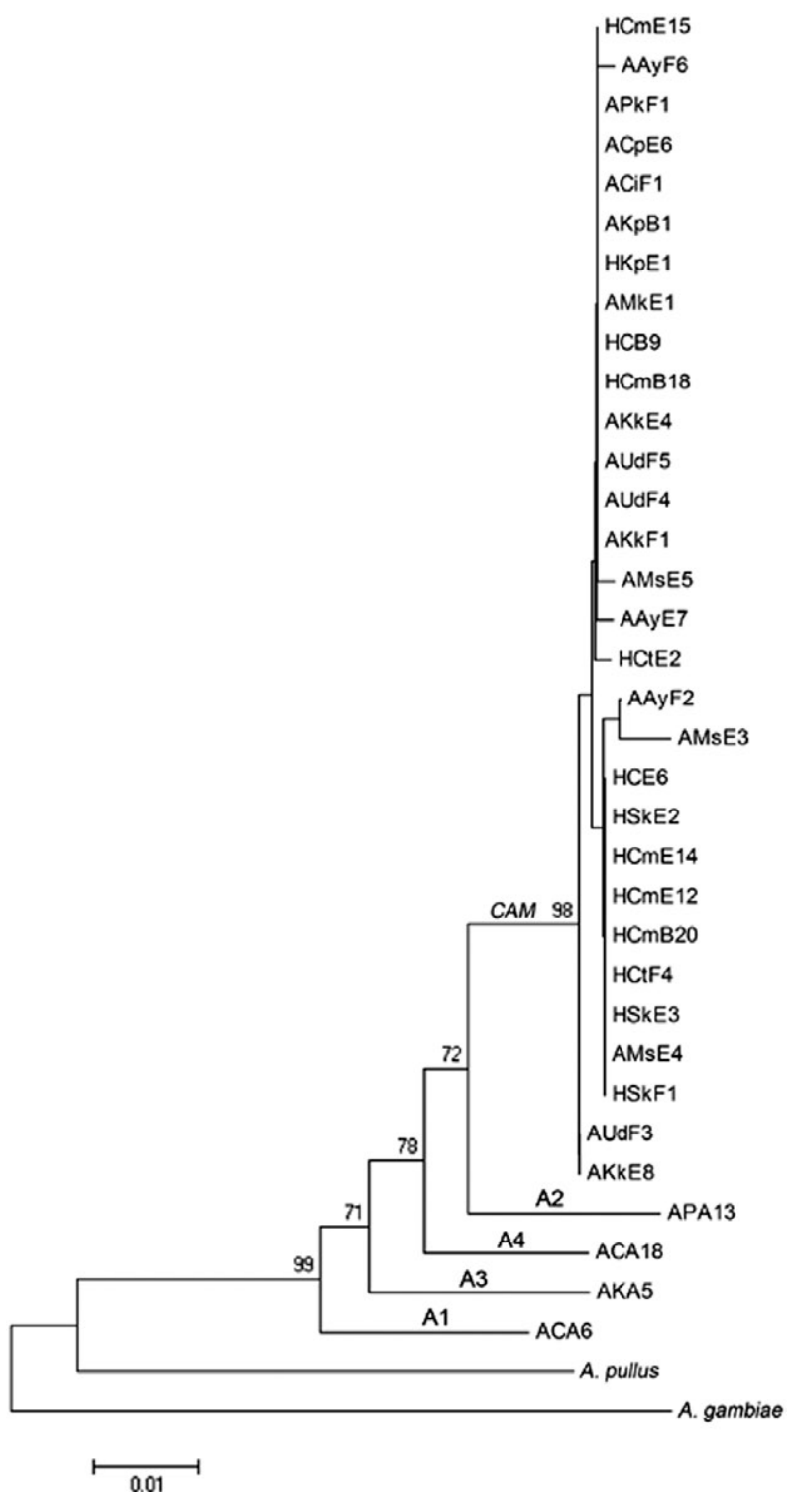

Fig. 5: a phylogenetic tree of Anopheles campestris-like Forms B, E and $\mathrm{F}(C A M)$ and Anopheles barbirostris species A1, A2, A3 and A4 based on molecular analysis of COII sequences. The tree was generated by neighbor-joining analysis. Numbers on the nodes indicate probabilities based on 1,000 bootstrap replicates. A probability of more than $50 \%$ is shown. Branch lengths are proportional to genetic distance (scale bar).

system are useful information to elucidate sibling species complexes in the Oriental Anopheles (Kanda et al. 1981, Baimai et al. 1987, Subbarao 1998). Nonetheless, a point worth noting is that an isoline colony established from the combinative characters of morphological, cytological (polytene and mitotic chromosomes) and/ or molecular markers must be seriously considered. A laboratory-raised colony established from a naturally mixed population should be omitted because it may be a mixture of cryptic species or sibling species (Subbarao 1998). Despite the differences in metaphase karyotypes 
TABLE II

Crossing experiments among the 12 isolines of Anopheles campestris-like Forms B, E and F

\begin{tabular}{|c|c|c|c|c|c|c|c|}
\hline \multirow{2}{*}{$\begin{array}{l}\text { Crosses } \\
\text { (female x male) }\end{array}$} & \multirow{2}{*}{$\begin{array}{l}\text { Total eggs } \\
\qquad(\mathrm{n})^{a}\end{array}$} & \multirow{2}{*}{$\begin{array}{l}\text { Embryonation } \\
\text { rate }^{b}\end{array}$} & \multirow{2}{*}{$\begin{array}{l}\text { Hatched } \\
\mathrm{n}(\%)\end{array}$} & \multirow{2}{*}{$\begin{array}{l}\text { Pupation } \\
\text { n }(\%)\end{array}$} & \multirow{2}{*}{$\begin{array}{c}\text { Emergence } \\
\mathrm{n}(\%)\end{array}$} & \multicolumn{2}{|c|}{$\begin{array}{c}\text { Total emergence } \\
(\%)\end{array}$} \\
\hline & & & & & & Female & Male \\
\hline \multicolumn{8}{|l|}{ Parental cross } \\
\hline HCE6 x HCE6 & $536(60,476)$ & 88 & $456(85.07)$ & 357 (78.29) & $336(94.12)$ & $162(48.21)$ & 174 (51.79) \\
\hline $\mathrm{AKpB} 1$ x AKpB1 & $577(286,291)$ & 85 & $433(75.04)$ & $312(72.06)$ & $303(97.12)$ & $154(50.83)$ & 149 (49.17) \\
\hline AAyF2 x AAyF2 & $484(186,298)$ & 71 & $335(69.21)$ & $256(76.42)$ & $242(94.53)$ & $142(58.68)$ & $100(41.32)$ \\
\hline AUdF5 x AUdF5 & $509(145,364)$ & 89 & $387(76.03)$ & 348 (89.92) & $306(87.93)$ & $156(50.98)$ & $150(49.02)$ \\
\hline $\mathrm{AKkE} 4 \times \mathrm{AKkE} 4$ & $499(171,328)$ & 95 & 455 (91.18) & $400(87.91)$ & $320(80.00)$ & $164(51.25)$ & $156(48.75)$ \\
\hline AMkE1 x AMkE1 & $515(202,313)$ & 84 & $443(86.02)$ & 363 (81.94) & $316(87.05)$ & $143(45.25)$ & $173(54.75)$ \\
\hline AMsE3 x AMsE3 & $469(186,283)$ & 75 & $352(75.05)$ & $299(84.94)$ & $250(83.61)$ & $110(44.00)$ & $140(56.00)$ \\
\hline ACiF1 x ACiF1 & $490(231,259)$ & 86 & 397 (81.02) & 381 (95.97) & 355 (93.18) & $153(43.10)$ & $202(56.90)$ \\
\hline HSkE3 x HSkE3 & $564(263,301)$ & 87 & $474(84.04)$ & $431(90.93)$ & $328(76.10)$ & $180(54.88)$ & $148(45.12)$ \\
\hline $\mathrm{HCtF} 4 \times \mathrm{HCtF} 4$ & $494(226,268)$ & 82 & $346(70.04)$ & $327(94.51)$ & $278(85.02)$ & $131(47.12)$ & $147(52.88)$ \\
\hline APkF1 x APkF1 & $525(197,328)$ & 93 & $389(74.10)$ & $319(82.01)$ & $290(90.91)$ & $130(44.83)$ & $160(55.17)$ \\
\hline ACpE6 x ACpE6 & $566(272,294)$ & 94 & $521(92.05)$ & 454 (87.14) & $427(94.05)$ & $217(50.82)$ & $210(49.18)$ \\
\hline \multicolumn{8}{|l|}{ Reciprocal cross } \\
\hline HCE6 x AKpB1 & $473(102,371)$ & 93 & $359(75.90)$ & 280 (77.99) & $258(92.14)$ & $130(50.39)$ & $128(49.61)$ \\
\hline AKpB1 x HCE6 & $360(87,273)$ & 74 & $220(61.11)$ & $216(98.18)$ & $190(87.96)$ & $96(50.53)$ & $94(49.47)$ \\
\hline HCE6 x AAyF2 & $467(170,297)$ & 71 & 309 (66.17) & $263(85.11)$ & $232(88.21)$ & $112(48.28)$ & $120(51.72)$ \\
\hline AAyF2 x HCE6 & $423(207,216)$ & 92 & $360(85.11)$ & $266(73.89)$ & $218(81.95)$ & $97(44.50)$ & $121(55.50)$ \\
\hline HCE6 x AUdF5 & $398(196,202)$ & 83 & $330(82.91)$ & $268(81.21)$ & $254(94.78)$ & $130(51.18)$ & $124(48.82)$ \\
\hline AUdF5 x HCE6 & $485(184,301)$ & 96 & $378(77.94)$ & 318 (84.13) & $309(97.17)$ & $151(48.87)$ & $158(51.13)$ \\
\hline HCE6 x AKkE4 & $402(171,231)$ & 94 & $382(95.02)$ & $330(86.39)$ & 307 (93.03) & $169(55.05)$ & $138(45.95)$ \\
\hline AKkE4 x HCE6 & $306(119,187)$ & 62 & $189(61.76)$ & $136(71.96)$ & 117 (86.03) & $63(53.85)$ & $54(46.15)$ \\
\hline HCE6 x AMkE1 & $499(186,313)$ & 95 & 459 (91.98) & 418 (91.07) & $372(89.00)$ & $179(48.12)$ & $193(51.88)$ \\
\hline AMkE1 x HCE6 & $424(138,286)$ & 86 & 344 (81.13) & 337 (97.97) & 317 (94.07) & $139(43.85)$ & $178(56.15)$ \\
\hline HCE6 x AMsE3 & $440(193,247)$ & 81 & $339(77.05)$ & 281 (82.89) & $235(83.63)$ & $120(51.06)$ & 115 (48.94) \\
\hline AAyF2 x HCE6 & $423(207,216)$ & 92 & $360(85.11)$ & $266(73.89)$ & $218(81.95)$ & $97(44.50)$ & $121(55.50)$ \\
\hline HCE6 x AUdF5 & $398(196,202)$ & 83 & $330(82.91)$ & $268(81.21)$ & $254(94.78)$ & $130(51.18)$ & $124(48.82)$ \\
\hline AUdF5 x HCE6 & $485(184,301)$ & 96 & $378(77.94)$ & 318 (84.13) & 309 (97.17) & $151(48.87)$ & $158(51.13)$ \\
\hline HCE6 x AKkE4 & $402(171,231)$ & 94 & $382(95.02)$ & $330(86.39)$ & 307 (93.03) & $169(55.05)$ & $138(45.95)$ \\
\hline AKkE4 x HCE6 & $306(119,187)$ & 62 & $189(61.76)$ & $136(71.96)$ & 117 (86.03) & $63(53.85)$ & $54(46.15)$ \\
\hline HCE6 x AMkE1 & $499(186,313)$ & 95 & 459 (91.98) & 418 (91.07) & $372(89.00)$ & $179(48.12)$ & $193(51.88)$ \\
\hline AMkE1 x HCE6 & $424(138,286)$ & 86 & 344 (81.13) & 337 (97.97) & $317(94.07)$ & $139(43.85)$ & $178(56.15)$ \\
\hline HCE6 x AMsE3 & $440(193,247)$ & 81 & $339(77.05)$ & $281(82.89)$ & $235(83.63)$ & $120(51.06)$ & 115 (48.94) \\
\hline APkF1 x HCE6 & $403(172,231)$ & 89 & $283(70.22)$ & $253(89.40)$ & 235 (92.89) & $133(56.60)$ & $102(43.40)$ \\
\hline HCE6 x ACpE6 & $383(33,350)$ & 90 & $306(79.90)$ & $301(98.37)$ & $247(82.06)$ & $114(46.15)$ & $133(53.85)$ \\
\hline ACpE6 x HCE6 & $473(159,314)$ & 99 & $445(94.08)$ & 414 (93.03) & $385(93.00)$ & $177(45.97)$ & $208(54.03)$ \\
\hline \multicolumn{8}{|l|}{$\mathrm{F}_{1}$ cross } \\
\hline $\begin{array}{l}(\mathrm{HCE} 6 \times \mathrm{AKpB1}) \mathrm{F}_{1} \\
\mathrm{x}(\mathrm{HCE} 6 \times \mathrm{AKpB} 1) \mathrm{F}_{1}\end{array}$ & $407(180,227)$ & 90 & 366 (89.93) & $278(75.96)$ & 231 (83.09) & $137(59.31)$ & $94(40.69)$ \\
\hline $\begin{array}{l}(\mathrm{AKpB} 1 \times \mathrm{HCE} 6)_{1} \\
\mathrm{x}(\mathrm{AKpB} 1 \times \mathrm{HCE} 6) \mathrm{F}_{1}\end{array}$ & $399(75,324)$ & 91 & 303 (75.94) & $251(82.84)$ & $226(90.04)$ & $104(46.02)$ & $122(53.98)$ \\
\hline $\begin{array}{l}(\mathrm{HCE} 6 \times \mathrm{AAyF} 2) \mathrm{F}_{1} \\
\mathrm{x}(\mathrm{HCE} 6 \times \mathrm{AAyF} 2) \mathrm{F}_{1}\end{array}$ & $529(226,303)$ & 81 & $397(75.05)$ & 369 (92.95) & 317 (85.91) & $158(49.84)$ & $159(50.16)$ \\
\hline $\begin{array}{l}(\text { AAyF2 x HCE6)F } \\
x\left(\text { AAyF2 } 2 \text { HCE6) } F_{1}\right.\end{array}$ & $541(224,317)$ & 86 & $422(78.00)$ & $301(71.33)$ & $265(88.04)$ & 135 (50.94) & $130(49.06)$ \\
\hline $\begin{array}{l}(\mathrm{HCE} 6 \times \mathrm{AUdF} 5)_{1} \\
\mathrm{x}(\mathrm{HCE} 6 \times \mathrm{AUdF}) \mathrm{F}_{1}\end{array}$ & $485(157,328)$ & 87 & 407 (83.92) & 338 (83.05) & 294 (86.98) & $141(47.96)$ & $153(52.04)$ \\
\hline $\begin{array}{l}(\mathrm{AUdF} 5 \times \mathrm{HCE} 6) \mathrm{F}_{1} \\
\mathrm{x}(\mathrm{AUdF} 5 \times \mathrm{HCE} 6) \mathrm{F}_{1}\end{array}$ & $438(159,279)$ & 81 & 350 (79.91) & 317 (90.57) & $282(88.96)$ & $127(45.04)$ & 155 (54.96) \\
\hline
\end{tabular}




\begin{tabular}{|c|c|c|c|c|c|c|c|}
\hline $\begin{array}{l}(\mathrm{HCE} 6 \times \mathrm{AKkE} 4) \mathrm{F}_{1} \\
\mathrm{x}(\mathrm{HCE} 6 \times \mathrm{AKkE} 4) \mathrm{F}_{1}\end{array}$ & $459(225,234)$ & 73 & 317 (69.06) & $288(90.85)$ & $265(92.01)$ & $138(52.08)$ & $127(47.92)$ \\
\hline $\begin{array}{l}(\mathrm{AKkE} 4 \times \mathrm{HCE} 6) \mathrm{F}_{1} \\
\mathrm{x}(\mathrm{AKkE} 4 \times \mathrm{HCE} 6) \mathrm{F}_{1}\end{array}$ & $400(136,264)$ & 77 & $280(70.00)$ & $262(93.57)$ & $215(82.06)$ & $109(50.70)$ & $106(49.30)$ \\
\hline $\begin{array}{l}(\mathrm{HCE} 6 \times \mathrm{AMkE} 1) \mathrm{F}_{1} \\
\mathrm{x}(\mathrm{HCE} 6 \times \mathrm{AMkE1}) \mathrm{F}_{1}\end{array}$ & $420(116,304)$ & 96 & $386(91.90)$ & $317(82.12)$ & $247(77.92)$ & $101(40.89)$ & $146(59.11)$ \\
\hline $\begin{array}{l}\left(\text { AMkE1 x HCE6)F } F_{1}\right. \\
\text { x }\left(\text { AMkE1 x HCE6) } F_{1}\right.\end{array}$ & $489(204,285)$ & 97 & 449 (91.82) & 332 (73.94) & $252(75.90)$ & $128(50.79)$ & $124(49.21)$ \\
\hline $\begin{array}{l}(\mathrm{HCE} 6 \times \mathrm{AMsE}) \mathrm{F}_{1} \\
\mathrm{x}(\mathrm{HCE} 6 \times \mathrm{AMsE}) \mathrm{F}_{1}\end{array}$ & $439(77,362)$ & 91 & 356 (81.09) & $263(73.88)$ & 228 (86.69) & $120(52.63)$ & $108(47.37)$ \\
\hline $\begin{array}{l}(\mathrm{AMsE} 3 \times \mathrm{HCE} 6) \mathrm{F}_{1} \\
\mathrm{x}(\mathrm{AMsE} 3 \times \mathrm{HCE} 6) \mathrm{F}_{1}\end{array}$ & $507(211,296)$ & 93 & 451 (88.95) & $343(76.05)$ & 281 (81.92) & $129(45.91)$ & $152(54.09)$ \\
\hline $\begin{array}{l}(\mathrm{HCE} 6 \times \mathrm{ACiF} 1) \mathrm{F}_{1} \\
\mathrm{x}(\mathrm{HCE} 6 \times \mathrm{ACiF} 1) \mathrm{F}_{1}\end{array}$ & $493(221,272)$ & 90 & $434(88.03)$ & 352 (81.11) & $250(71.02)$ & $132(52.80)$ & $118(47.20)$ \\
\hline $\begin{array}{l}(\mathrm{ACiF} 1 \mathrm{x} \text { HCE6)F } \\
\text { x }(\mathrm{ACiF} 1 \times \mathrm{HCE} 6) \mathrm{F}_{1}\end{array}$ & $491(201,290)$ & 79 & 349 (71.08) & $293(83.95)$ & $252(86.01)$ & $116(46.03)$ & $136(53.97)$ \\
\hline $\begin{array}{l}\left(\mathrm{HCE} 6 \mathrm{x} \mathrm{HSkE3} \mathrm{F}_{1}\right. \\
\mathrm{x}\left(\mathrm{HCE} 6 \mathrm{x} \mathrm{HSkE3} \mathrm{F}_{1}\right.\end{array}$ & $443(154,289)$ & 89 & $381(86.00)$ & 312 (81.89) & $275(88.14)$ & $140(50.91)$ & $135(49.09)$ \\
\hline $\begin{array}{l}\left(\text { HSkE3 } x \text { HCE6)F } F_{1}\right. \\
\text { x (HSkE3 x HCE6)F } F_{1}\end{array}$ & $531(249,282)$ & 98 & 494 (93.03) & 449 (90.89) & $350(77.95)$ & $161(46.00)$ & $189(54.00)$ \\
\hline $\begin{array}{l}(\mathrm{HCE} 6 \times \mathrm{HCtF} 4) \mathrm{F}_{1} \\
\mathrm{x}(\mathrm{HCE} 6 \times \mathrm{HCtF} 4) \mathrm{F}_{1}\end{array}$ & $374(101,273)$ & 93 & 359 (95.99) & $330(91.92)$ & $271(81.12)$ & $146(53.87)$ & $125(46.13)$ \\
\hline $\begin{array}{l}(\mathrm{HCtF} 4 \times \mathrm{HCE} 6) \mathrm{F}_{1} \\
\mathrm{x}(\mathrm{HCtF} 4 \times \mathrm{HCE} 6) \mathrm{F}_{1}\end{array}$ & $424(79,345)$ & 86 & 399 (94.10) & 391 (97.99) & $305(78.01)$ & $135(44.26)$ & $170(55.74)$ \\
\hline $\begin{array}{l}(\mathrm{HCE} 6 \times \mathrm{APkF} 1) \mathrm{F}_{1} \\
\mathrm{x}(\mathrm{HCE} 6 \times \mathrm{APkF} 1) \mathrm{F}_{1}\end{array}$ & $506(227,279)$ & 80 & $380(75.10)$ & 331 (87.11) & $245(74.02)$ & $118(48.16)$ & $127(51.84)$ \\
\hline $\begin{array}{l}\text { (APkF1 x HCE6)F } F_{1} \\
\text { x (APkF1 x HCE6)F } F_{1}\end{array}$ & $531(259,272)$ & 92 & 431 (81.17) & 344 (79.81) & $276(80.23)$ & 141 (51.09) & 135 (48.91) \\
\hline $\begin{array}{l}(\mathrm{HCE} 6 \times \mathrm{ACpE} 6) \mathrm{F}_{1} \\
\text { x }(\mathrm{HCE} 6 \times \mathrm{ACpE} 6) \mathrm{F}_{1}\end{array}$ & $545(269,276)$ & 91 & 447 (82.02) & 387 (86.58) & 325 (83.98) & $136(41.85)$ & $189(58.15)$ \\
\hline $\begin{array}{l}\left(\text { ACpE6 x HCE6) } F_{1}\right. \\
x\left(\text { ACpE6 x HCE6) } F_{1}\right.\end{array}$ & $397(69,328)$ & 89 & 346 (87.15) & $243(70.23)$ & 224 (92.18) & $106(47.32)$ & $118(52.68)$ \\
\hline
\end{tabular}

$a$ : two selective egg-batches of inseminated females from each cross; $b$ : dissection from 100 eggs.

of An. campestris-like Forms B, E and F, either from sympatric or allopatric populations, the present studies revealed no post-mating reproductive isolation among the three karyotypic forms. This is in contrast to the case of the An. barbirostris complex in which all four sibling species exhibited distinct metaphase karyotypes, particularly the sex chromosomes (Saeung et al. 2008, Suwannamit et al. 2009).

Molecular investigations of some specific genomic markers, e.g., rDNA (ITS1, ITS2, D3) and mtDNA (COI and COII) have been used extensively as a supportive tool to determine and/or characterise sibling species or cryptic species of anopheline mosquitoes (Mitchell et al. 1992, Sharpe et al. 2000, Min et al. 2002, Park et al. 2003, Junkum et al. 2005, Saeung et al. 2007, 2008). The molecular evidence of very low intraspecies variation (genetic distance $<0.005$ ) of ITS2 of rDNA and COI and COII of mtDNA among the 30 isolines of $\mathrm{An}$. campestris-like Forms B, E and F strongly supports a conspecific nature of these karyotypic forms. Therefore, we can confidently conclude that An campestris-like Forms $\mathrm{B}, \mathrm{E}$ and $\mathrm{F}$ represent intraspecies karyotypic variation due to the gain of heterochromatin in sex chromosomes in Thai populations. Similar results have been reported in other Asian anopheline mosquitoes, e.g., Anopheles sinensis Forms A and B (Choochote et al. 1998, Min et al. 2002), Anopheles vagus Forms A and B (Choochote et al. 2002), An. pullus Forms A and B (Park et al. 2003), Anopheles aconitus Forms B and C (Junkum et al. 2005) and South American anopheline mosquitoes, e.g., Anopheles darlingi and Anopheles nuneztovari (Rafael \& Tadei 1998, 2000) and Anopheles albitarsis (Rafael et al. 2005). Such heterochromatin variation in sex chromosomes is a general phenomenon in Anopheles and some dipteran insects (Baimai 1998). Moreover, the results in phylogenetic analysis based on the rDNA ITS2 sequences clearly support previous findings, suggesting that $A n$. campestris-like is more closely related to An. barbirostris species A4 than to species A1, A2 and A3 (Suwannamit et al. 2009). In this study, the genetic distances between An. campestris-like and An. barbirostris species A2 are close to those between An. campestris-like and An. barbirostris species A4. Therefore, our study suggested that $A n$. campestris-like is more closely related 


\section{TABLE III}

Average genetic distance within and between the Anopheles campestris-like

Forms B, E and F for the ITS2, COI and COII regions

\begin{tabular}{lccc}
\hline & ITS2 & COI & COII \\
\hline Within Form & & & \\
B & 0.001 & 0.004 & 0.001 \\
E & 0.002 & 0.004 & 0.002 \\
F & 0.002 & 0.003 & 0.002 \\
Between Forms & & & \\
B-E & 0.002 & 0.004 & 0.001 \\
B-F & 0.002 & 0.004 & 0.001 \\
E-F & 0.002 & 0.003 & 0.002 \\
\hline
\end{tabular}

to An. barbirostris species A2 and A4 than to species A1 and A3 for COI and COII. Additionally, the crossing experiments also supported molecular evidence because the reciprocal crosses between An. campestris-like Form $\mathrm{E}$ and $A n$. barbirostris species $\mathrm{A} 4$ yielded $\mathrm{F}_{1}$-hybrids in both directions, with lower degrees of asynaptic polytene chromosomes than those for the crosses A1 x A4, A 2 x A4 and A3 x A4 (Suwannamit et al. 2009). Further detailed investigation of population biology for these sibling species may shed some light on speciation processes of these anopheline mosquitoes in Thailand.

\section{ACKNOWLEDGEMENTS}

To Dr. J. Milne, for valuable suggestions on the draft manuscript, and to Dr. Niwes Nantachit, Dean of the Faculty of Medicine, Chiang Mai University, for his interest in this research project.

\section{REFERENCES}

Atomosoedjono S, van Peenen PF, Putrali J 1976. Anopheles barbirostris (Van der Wulp) still an efficient vector of Brugia malayi in Central Sulawesi (Celebes), Indonesia. Trans R Soc Trop Med Hyg 70: 259.

Baimai V 1998. Heterochromatin accumulation and karyotypic evolution in some dipteran insects. Zool Stud 37: 75-88.

Baimai V, Andre RG, Harrison BA, Kijchalao U, Panthusiri L 1987. Crossing and chromosomal evidence for two additional sibling species within the taxon Anopheles dirus Peyton and Harrison (Diptera: Culicidae) in Thailand. Proc Entomol Soc Wash 89: 157-166.

Baimai V, Rattanarithikul R, Kijchalao U 1995. Metaphase karyotypes of Anopheles of Thailand and Southeast Asia: IV. The barbirostris and umbrosus species groups, subgenus Anopheles (Diptera: Culicidae). J Am Mosq Control Assoc 11: 323-328.

Choochote W, Jitpakdi A, Rongsriyam Y, Komalamisra N, Pitasawat $\mathrm{B}$, Palakul K 1998. Isoenzyme study and hybridization of two forms of Anopheles sinensis (Diptera: Culicidae) Northern Thailand. Southeast Asian J Trop Med Public Health 29: 841-848.

Choochote W, Jitpakdi A, Sukontason KL, Chaithong U, Wongkamchai S, Pitasawat B, Jariyapan N, Suntaravitun T, Rattanachanpichai E, Sukontason K, Leemingsawat S, Rongsriyam Y 2002. Intraspecific hybridization of two karyotypic forms of Anopheles vagus (Diptera: Culicidae) and the related egg surface topography. Southeast Asian J Trop Med Public Health 33 (Suppl. 3): 29-35.
Choochote W, Sucharit S, Abeywickreme W 1983. Experiments in crossing two strains of Anopheles barbirostris Van der Wulp 1884 (Diptera: Culicidae) in Thailand. Southeast Asian J Trop Med Public Health 14: 204-209.

Griffith ME 1955. A note on Anopheles minimus Theobald as a malaria vector in Thailand. VI Intern Congr Microbiol 5: 565-567.

Harbach RE 2004. The classification of genus Anopheles (Diptera: Culicidae): a working hypothesis of phylogenetic relationships. Bull Entomol Res 94: 537-553.

Harrison BA 1980. Medical entomology studies: XIII. The Myzomyia series of Anopheles (Cellia) in Thailand, with emphasis on intrainterspecific variations (Diptera: Culicidae). Contrib Am Entomol Inst 17: 1-195.

Iyengar MOT 1953. Filariasis in Thailand. Bull WHO 9: 731-766.

Junkum A, Komalamisra N, Jitpakdi A, Jariyapan N, Min GS, Park MH, Cho KH, Somboon P, Bates PA, Choochote W 2005. Evidence to support two conspecific cytological races of Anopheles aconitus in Thailand. $J$ Vector Ecol 30: 213-224.

Kanda T, Takai K, Chiang GL, Cheong WH, Sucharit S 1981. Hybridization and some biological facts of seven strains of the Anopheles leucosphyrus group (Reid, 1968). Jpn J Sanit Zool 32: 321-329.

Kim SJ, Choochote W, Jitpakdi A, Junkum A, Park SJ, Min GS 2003. Establishment of a self-mating mosquito colony of Anopheles sinensis from Korea. Korean J Entomol 33: 267-271.

Kimura M 1980. A simple method for estimating evolutionary rates of base substitution through comparative studies of nucleotide sequences. J Mol Evol 16: 111-120.

Kirnowardoyo S 1985. Status of Anopheles malaria vectors in Indonesia. Southeast Asian J Trop Med Pub Health 16: 129-132.

Limrat D, Rojruthai B, Apiwathnasorn C, Samung Y, Prommongkol S 2001. Anopheles barbirostris/campestris as a probable vector of malaria in Aranyaprathet, Sa Kaeo province. Southeast Asian J Trop Med Public Health 32: 739-744.

Min GS, Choochote W, Jitpakdi A, Kim SJ, Kim W, Jung J, Junkum A 2002. Intraspecific hybridization of Anopheles sinensis (Diptera: Culicidae) strains from Thailand and Korea. Mol Cells 14: 198-204.

Mitchell SE, Narang SK, Cockburn AF, Seawright JA, Goldenthal M 1992. Mitochondrial and ribosomal DNA variation among members of the Anopheles quadrimaculatus (Diptera: Culicidae) species complex. Genome 35: 939-950.

Park SJ, Choochote W, Jitpakdi A, Junkum A, Kim SJ, Jariyapan N, Park JW, Min GS 2003. Evidence for a conspecific relationship between two morphologically and cytologically different Forms of Korean Anopheles pullus mosquito. Mol Cells 16: 354-360.

Rafael MS, Santos-Junior IP, Tadei WP, Sallum MA, Forattini OP 2005. Karyotype of Brazilian Anopheles albitarsis sensu lato (Diptera: Culicidae). Genet Mol Res 4: 684-690.

Rafael MS, Tadei WP 1998. Metaphase karyotypes of Anopheles (Nyssorhynchus) darlingi Root and An. (N.) nuneztovari GabaldÓn (Diptera: Culicidae). Genet Mol Biol 21: 351-354.

Rafael MS, Tadei WP 2000. Heterochromatin variation in chromosomes of Anopheles (Nyssorhynchus) darlingi Root and An. (N.) nuneztovari GabaldÓn (Diptera: Culicidae). Genet Mol Biol 23: 67-70.

Rattanarithikul R, Harrison BA, Harbach RE, Panthusiri P, Coleman RE 2006. Illustrated keys to the mosquitoes of Thailand IV Anopheles. Southeast Asian J Trop Med Public Health 37 (Suppl. 2): 1-128.

Reid JA 1968. Anopheline mosquitoes of Malaya and Borneo. Stud Inst Med Res Malaya 31: 1-520. 
Saeung A, Baimai V, Otsuka Y, Rattanarithikul R, Somboon P, Junkum A, Tuetun B, Takaoka H, Choochote W 2008. Molecular and cytogenetic evidence of three sibling species of the Anopheles barbirostris Form A (Diptera: Culicidae) in Thailand. Parasitol Res 102: 499-507.

Saeung A, Otsuka Y, Baimai V, Somboon P, Pitasawat B, Tuetun B, Junkum A, Takaoka H, Choochote W 2007. Cytogenetic and molecular evidence for two species in the Anopheles barbirostris complex (Diptera: Culicidae) in Thailand. Parasitol Res 101: 1337-1344.

Saitou N, Nei M 1987. The neighbor-joining method: a new method for reconstructing phylogenetic trees. Mol Biol Evol 4: 406-425.

Sattabongkot J, Tsuboi T, Zollner GE, Sirichaisinthop J, Cui L 2004. Plasmodium vivax transmission: chances for control? Trends Parasitol 20: 192-198.

Scanlon JE, Peyton EL, Gould DJ 1968. An annotated checklist of the Anopheles of Thailand. Thai Natl Sci Pap Fauna Ser 2: 1-35.
Sharpe RG, Harbach RE, Butlin RK 2000. Molecular variation and phylogeny of members of the Minimus group of Anopheles subgenus Cellia (Diptera: Culicidae). Syst Entomol 25: 263-272.

Subbarao SK 1998. Anopheline species complexes in South-East Asia, WHO Tech Pub Ser, Geneva, Vol. XVIII, 82 pp.

Suwannamit S, Baimai V, Otsuka Y, Saeung A, Thongsahuan S, Tuetun B, Apiwathnasorn C, Jariyapan N, Somboon P, Takaoka H, Choochote W 2009. Cytogenetic and molecular evidence for an additional new species within the taxon Anopheles barbirostris (Diptera: Culicidae) in Thailand. Parasitol Res 104: 905-918.

Tamura K, Dudley J, Nei M, Kumar S 2007. MEGA4: molecular evolution genetics analysis (MEGA) software version 4.0. Mol Biol Evol 24: 1596-1599.

Thompson JD, Higgins DG, Gibson TJ 1994. CLUSTAL W: improving the sensitivity of progressive multiple sequence alignment through sequence weighting, positions-specific gap penalties and weight matrix choice. Nucleic Acids Res 22: 4673-4680. 